\title{
Filamentous Bacteriophage Delay Healing of Pseudomonas-Infected Wounds
}

Michelle S. Bach ${ }^{1,2 \#}$, Christiaan R. de Vries ${ }^{1 \#, ~ J o h a n n a ~ M . ~ S w e e r e ~}{ }^{1,2}$, Medeea Popescu ${ }^{1,2}$, Jonas D. Van Belleghem $^{1}$, Gernot Kaber ${ }^{1}$, Elizabeth B. Burgener ${ }^{3}$, Dan Liu ${ }^{1}$, Quynh-Lam Tran ${ }^{1}$, Tejas Dharmaraj ${ }^{1}$, Maria Birukova $^{1,2}$, Vivekananda Sunkari ${ }^{1,2}$, Swathi Balaji ${ }^{4}$, Sundeep Keswani ${ }^{4}$, Niaz Banaei ${ }^{5}$, Dolly K. Khona ${ }^{6}$, Laurence Nedelec ${ }^{1}$, Chandan K. Sen ${ }^{6}$, Venita Chandra7, Patrick R. Secor ${ }^{8}$, Gina A. Suh, ${ }^{9 \dagger}$ and Paul L. Bollyky ${ }^{1+*}$

\section{Affiliations:}

${ }^{1}$ Division of Infectious Diseases and Geographic Medicine, Department of Medicine, Stanford University, Stanford, CA

${ }^{2}$ Stanford Immunology, Stanford University, Stanford, CA.

${ }^{3}$ Center for Excellence in Pulmonary Biology, Department of Pediatrics, Stanford University, Stanford, CA

${ }^{4}$ Division of Pediatric Surgery, Department of Surgery, Baylor College of Medicine, Houston, TX

${ }^{5}$ Division of Pathology, Department of Medicine, Stanford University, Stanford, CA

${ }^{6}$ Department of Surgery, Indiana University, Indianapolis, IN

${ }^{7}$ Department of Vascular Surgery, Stanford University, Stanford, CA

${ }^{8}$ University of Montana, Missoula, MT

${ }^{9}$ Division of Infectious Diseases, Mayo Clinic, Rochester, MN

\# Contributed equally to the work

$\dagger$ These authors share senior authorship.

* To whom correspondence should be addressed: pbollyky@stanford.edu. Twitter: @ bollyky 


\begin{abstract}
$\underline{\text { Abstract }}$
We have identified a novel role for filamentous bacteriophage in the delayed healing associated with chronic Pseudomonas aeruginosa $(\mathrm{Pa})$ wound infections. In a mouse model of chronic $\mathrm{Pa}$ - infected wounds, Pf, a filamentous phage produced by $P a$, impaired keratinocyte migration, prevented wound reepithelialization, and delayed healing in both the absence and presence of live bacteria. Mechanistically, the immune response to Pf phage produces soluble factors that impair keratinocyte migration and delay wound re-epithelialization. In a prospective cohort study of 113 human patients, $P a$ was detected in 36 patients and 25 of these (69\%) were positive for $\operatorname{Pf}$ phage. $\operatorname{Pf}(+)$ wounds were significantly older and more likely to increase in size over time than Pf(-) wounds. Together, these data implicate Pf in the delayed wound healing associated with $\mathrm{Pa}$ infection. We propose that Pf phage may have potential as a biomarker and therapeutic target for delayed wound healing.
\end{abstract}

Word Count: 147 words

Keywords: bacteriophage, wounds, microbiology, immunology, Pseudomonas, Pf 


\section{$\underline{\text { Introduction }}$}

Chronic wounds are associated with extensive human suffering and massive economic costs (Järbrink et al., 2016; Kirsner, 2016). Over 6.5 million Americans have chronic wounds, and their care is estimated to cost $\$ 25$ billion annually (Kirsner, 2016). Bacterial infections frequently complicate chronic wounds, leading to delayed wound healing (Guo and Dipietro, 2010), increased rates of amputation (Gardner and Frantz, 2008), and extensive morbidity and mortality (Bowler, 2002; Clinton and Carter, 2015; Scali and Kunimoto, 2013).

Wounds infected with the Gram-negative pathogen Pseudomonas aeruginosa ( $\mathrm{Pa}$ ) are characterized by poor outcomes (Dowd et al., 2008; James et al., 2008; Kirketerp-Møller et al., 2008; Malic et al., 2009). $P a$ is prevalent in infections of burns (Tredget et al., 2004), diabetic ulcers, and post-surgical sites (Percival et al., 2015). The presence of $P a$ is associated with delayed wound closure in both humans as well as animal models (Bryers, 2008; Guo and Dipietro, 2010; Watters et al., 2013; Zhao et al., 2012), and wounds infected with $P a$ tend to be larger than those in which $P a$ is not detected (Gjødsbøl et al., 2006; Jesaitis et al., 2003). This wound chronicity is associated with ineffective bacterial clearance (Sen et al., 2009) and delayed wound healing (Bjarnsholt et al., 2008). In addition to wound infections, $P a$ is a major human pathogen in other contexts (Bryers, 2008; Høiby et al., 2011; Sen et al., 2009) due to increased antibiotic resistance incidence. In recognition of the magnitude of this problem, $P a$ was recently listed as a critical priority pathogen by the World Health Organization (WHO) and the Centers for Disease Control (CDC)(Centers for Disease Control and, 2013; Tacconelli et al., 2018). Therefore, there is great interest in identifying novel biomarkers, virulence factors, and therapeutic targets associated with $\mathrm{Pa}$ infections.

One such potential target is $\mathrm{Pf}$ bacteriophage (Pf), a filamentous virus produced by $\mathrm{Pa}$ (Dowd et al., 2008; Malic et al., 2009). Unlike lytic bacteriophages used in phage therapy (Gordillo Altamirano and Barr, 2019; Górski et al., 2019), Pf typically does not lyse its bacterial hosts. Instead, Pf phages integrate into the bacterial chromosome as prophage and can be produced without destroying their bacterial hosts (Rakonjac et al., 2011; Roux et al., 2019). Indeed, the production of phage virions is partially under bacterial control (Castang and Dove, 2012). We and others have reported that Pf phage contribute to $P a$ fitness by 
serving as structural elements in $\mathrm{Pa}$ biofilms (Secor et al., 2015b) and contributing to bacterial phenotypes associated with chronic $\mathrm{Pa}$ infection, including aggregation (Secor et al., 2018) and reduced motility (Secor et al., 2017).

Pf phage also impact host immunity in ways that promote chronic infection. We recently reported that Pf phage directly alter cytokine production and decrease phagocytosis by macrophages (Secor et al., 2017; Sweere et al., 2019b). These effects were associated with endocytosis of Pf by mammalian cells and triggering of maladaptive anti-viral immune responses that antagonize anti-bacterial immunity. Consistent with these effects, $P a$ strains that produced Pf phage ( $\operatorname{Pf}(+)$ strains) were more likely than strains that do not produce $\operatorname{Pf}(\operatorname{Pf}(-)$ strains) to establish wound infections in mice (Sweere et al., 2019b). These and other studies implicate Pf phage as a virulence factor in $\mathrm{Pa}$ infections (Burgener et al., 2019; Rice et al., 2009; Secor et al., 2015b).

We hypothesized that Pf phage might contribute to the delayed wound healing often associated with $P a$ wound infections. To test this, we first examined the effects of Pf phage in a well-established in vitro wound healing model (a scratch assay). We then examined the effects of Pf phage on wound healing in an in vivo mouse model in the absence or presence of live $P a$ (Sweere et al., 2019a; Sweere et al., 2019b). Finally, we examined the association between Pf phage and delayed wound healing in a cohort of 113 patients seen at the Stanford Advanced Wound Care Center. 


\section{$\underline{\text { Results }}$}

Infection with a $\mathrm{Pf}(+)$ strain of $P a$ causes worse morbidity in a murine chronic wound infection model.

We first examined the impact of a Pf phage-positive (Pf+) strain of $\mathrm{Pa}$ (PAO1) versus a Pf phagenegative (Pf-) isogenic strain (PAO1 $\Delta \mathrm{Pf} 4)$ on morbidity and mortality in a delayed inoculation murine wound infection model in which a provisional wound matrix is allowed to form for 24 hours after wounding before introduction of bacteria. We previously pioneered this model to study the initial establishment of $P a$ wound infections in otherwise healthy C57BL/6 mice in the absence of foreign material, diabetes, or other forms of immune suppression (de Vries et al.; Sweere et al., 2019a; Sweere et al., 2019b). Here, we have adapted this model to study wound healing in the setting of infection over a two-week time period. In brief, we generated bilateral, full-thickness, excisional wounds on the dorsum of mice. These wounds were then inoculated 24 hours later with $1 \times 10^{7} / \mathrm{mL}$ PAO1, PAO1 $\triangle \mathrm{Pf} 4$, or PBS as a control. We then assessed wound size daily through day 13 post- $P a$ inoculation (day 14 post-wounding). A schematic of this protocol is shown in Figure 1A.

All mice survived the 14-day experiment (Figure S1) and all wounds were obviously infected on Day 3 by gross appearance (Figure 1B), regardless of whether they received PAO1 or PAO1 $\Delta \mathrm{Pf} 4$. However, wounds in the PAO1 group were characterized by diffuse inflammation and disintegration of the epithelium of the wounded area. In contrast, PAO1 $\Delta \mathrm{Pf} 4$ wounds were characterized by less extensive inflammation and redness limited to the area around the wounded area (Figure 1B). Moreover, mice inoculated with PAO1 lost significant weight compared to mice that received PAO1 $\triangle \mathrm{Pf} 4$ or PBS (Figure 1C). These data implicate Pf phage in worse morbidity in this model.

\section{Pf phage delay wound healing.}

We next examined the effect of Pf phage on wound healing in our in vivo model. Healing was defined as the remaining open wound area as a fraction of the original wound area. Inoculation with PAO1 $\Delta \mathrm{Pf} 4$ led to significantly improved wound healing over time compared to inoculation with PAO1. Indeed, wound healing in many of the mice inoculated with PAO1 appeared "stuck" and mice failed to resolve their wounds 
(Figure 1D). By Day 13, most of the mice from the PAO1 $\triangle \mathrm{Pf} 4$ group resolved their infections and healed their wounds completely, whereas most wounds infected with PAO1 persisted (Figure 1E). Similar results were observed when area under curve (AUC), a measure of cumulative change in wound area, was considered (Figure 1F).

We next examined wound histology from tissue sections collected on Day 13 post-wounding that were collected from these mice. We observed that wounds infected with PAO1 $\Delta \mathrm{Pf} 4$ exhibited healthy granulation tissue (Figure 1G) whereas wounds infected with PAO1 had substantial necrotic debris, minimal granulation tissue, and obvious infection (Figure 1H). When the epithelial gap was measured, wounds infected with PAO1 were found to have significantly less re-epithelialization by day 13 compared to PAO1 $\Delta \mathrm{Pf} 4$ (Figure 1I).

Taken together, these data indicate that the production of Pf phage by $\mathrm{Pa}$ is associated with increased morbidity, ongoing infection, impaired wound healing, and delayed re-epithelialization in this model.

\section{Pf phage delay re-epithelialization in the absence of bacterial infection.}

Because the aforementioned data were collected in the setting of infection, it was difficult to know what to attribute to ongoing infection versus direct effect of Pf on wound healing. To assess this directly, we treated mice with purified Pf phage in the absence of bacterial infection. In particular, we administered heat killed PAO1 (HK-Pa) or PBS to C57BL6 mice one day after wounding in the absence or presence of supplemental Pf4 phage. Day 7 rather than Day 13 was used in the studies in Figure 2 because wounds healed more rapidly in the absence of live bacterial infections. Moreover, because in the absence of infection mouse wounds heal with contracture we used a standard splinting procedure that incorporates silicone rings to prevent this (Wang et al., 2013). A schematic detailing this protocol is shown in Figure 2A. Both the wound area on Day 1 (Figure 2B) and the epithelial gap on Day 7 post-wounding (Figure 2C) were assessed morphometrically. Representative area demarcations are shown inset into these images. 
We observed that supplementation with Pf4 phage was associated with a larger epithelial gap on Day 7 when normalized to Day 1 wound area (Figure 2D). However, overall inflammatory cell counts were not altered by Pf phage supplementation (Figure S2). We conclude that supplementation with Pf phage reduced re-epithelialization of murine wounds in the absence of infection.

\section{Pf phage enhance in vitro Pa biofilm formation.}

We next investigated the effects of Pf phage on biofilm structure, given the importance of this in chronic wounds and delayed healing (Zhao et al., 2013). We seeded PAO1 and PAO1 $\triangle \mathrm{Pf} 4$ on polycarbonate membrane discs and stained them with Sypro Ruby (biofilm matrix stain) and Syto9 (cell stain). Confocal laser scanning microscopy showed significantly increased biofilm thickness of PAO1 compared to PAO1 $\Delta$ Pf4 (Figure S3A-B). Supporting this result, microtiter plate crystal violet assay for biofilm attachment showed a significantly increased absorbance at 24 hours in PAO1 compared to PAO1 $\Delta \mathrm{Pf} 4$ (Figure S3C). Finally, scanning electron microscopy (SEM) of $P a$ cultures on membrane discs showed enhanced biofilm formation in PAO1 compared to PAO1 $\triangle \mathrm{Pf} 4$ (Figure S3D). Together, these data indicate that Pf phage promote formation of more robust biofilms.

\section{Supernatant from macrophages exposed to Pf phage impair in vitro keratinocyte migration}

We next asked whether Pf phage inhibit cell migration in a scratch assay — an in vitro model commonly used to study mechanisms related to wound healing (Liang et al., 2007). A schematic of this protocol is shown with representative examples of keratinocyte migration over 24 hours (Figure 3A).

Migration of HaCaT keratinocytes was not affected by purified Pf phage (Figure 3B). Although a few ( 1\%) HaCaT cells take up AlexaFluor 488-labeled Pf phage (Figure 3C) this is a negligible amount compared to the relatively large numbers of monocytes and macrophages that we previously reported take up Pf both in vitro and within the wound bed (Sweere et al., 2019b).

Given our previous reports that internalization of Pf phage suppresses production of TNF $\alpha$ made in response to LPS (Sweere et al., 2019b) as well as a number of other chemokines and cytokines (Secor et 
al., 2017), we hypothesized that changes in the wound inflammatory milieu might indirectly impact keratinocyte migration and thus wound healing. In support of this model, we found that supernatant from U937 macrophages stimulated with Pf and LPS slowed migration of HaCaT keratinocytes compared to supernatant from keratinocytes cultured in supernatant from mock controls (Figure 3D-E). U937 macrophages stimulated with Pf with or without LPS showed increased uptake of Pf compared to controls (Figure 3F). These data point to an indirect role for Pf phage in inhibition of keratinocyte migration. Consistent with this, we previously reported that macrophages as well as a number of other cell types take up Pf phage within the wound bed (Sweere et al., 2019b).

\section{Pf phage alter macrophage cytokine production profiles.}

To better understand how macrophage internalization of Pf phage impacts cytokine and chemokine production we performed a Luminex assay on primary human monocytes stimulated with Pf phage and LPS. We find that murine bone marrow-derived macrophages (BMDMs) take up Pf phage (Figure 4A) and that this leads to altered production of multiple cytokines and chemokines, including TNF (Figure 4B) as well as MIG (CXCL9), SDF1a (CXCL12), NGF (nerve growth factor), and IL-10 (Figure 4C).

We then attempted to overcome the inhibitory effects of Pf phage on keratinocyte migration and wound healing in mice via supplementation with several factors shown here or in our previous studies to be suppressed by Pf phage, including TNF, CXCL1, or IL-10 (Secor et al., 2017; Sweere et al., 2019b). However, these agents did not overcome the effect of Pf or restore normal wound healing in this model (data not shown).

Together these data suggest that Pf phage alter the inflammatory milieu in ways that impact keratinocyte migration and re-epithelialization. Further, these effects are either multi-factorial or involve cytokines and chemokines other than those directly tested here.

\section{Pf phage are abundant in human wounds infected with $P$. aeruginosa}


In light of the decreased wound healing observed in the presence of Pf4 phage in our in vivo model, we investigated the clinical impact of Pf phage on human chronic $P a$ wound infections. A total of 113 consecutive patients referred to the Infectious Disease service at the Stanford Advanced Wound Care Center (AWCC) were enrolled from June 2016 to June 2018. Our protocol for screening and classifying these patients is described in Figure S4. Patient characteristics are described in Table 1. Three Pa-positive patients (one who was $\operatorname{Pf}(+)$,and two who were $\operatorname{Pf}(-)$ ) were excluded from the final analysis due to amputation, exposed bone/tendon, or loss to follow up (Figure S4, S5). Microbiological characteristics of the $P a$-positive wound isolates are described in Table 2. Representative images from $P a$-infected $\operatorname{Pf}(+)$ and Pf(-) wounds are shown in Figure 5A,B. Additional representative images are shown in Figure S6.

We found that samples from 39 unique patients out of 113 were Pa-positive (35\%) (Figure 5C). Of the $\mathrm{Pa}$-positive patients included in our analysis, Pf prophage (integrated into the bacterial chromosome) was detected in $69 \%(25 / 36)$ of the wounds. Pf phage levels ranged from $3.55 \times 10^{3}-2.69 \times 10^{8}$ copies/swab, with a mean of $2.16 \times 10^{7}$ copies/swab in $\operatorname{Pf}(+)$ samples. Subjects in the $\mathrm{Pf}(-)$ subset were older compared to the $\mathrm{Pf}(+)$ subset $(61.6+/-14.7$ versus $76.6+/-14.5 ; \mathrm{p}=0.008$; Table 1). Higher numbers of $\operatorname{Pf}(+)$ patients were on antimicrobial treatments compared to $\operatorname{Pf}(-)$ patients, though this was not statistically significant (56\% vs 18\%; $\mathrm{p}=0.067$; Table 2 ). There were no significant correlations between Pf phage status and gender, BMI, recurrence of infection, race/ethnicity, co-morbidities, antimicrobial resistance, or co-infection with other bacterial or fungal species (Table 2). This excludes co-infection as a confounding variable and highlights the correlation between Pf phage status and clinical outcomes.

\section{Pf phage are associated with chronic $P a$ wound infections}

We assessed whether the absence or presence of Pf phage was associated with chronic or nonhealing human wound infections, as characterized by wound age. Chronic wounds are defined as lasting longer than 3 months in duration without complete healing (32). We therefore asked whether chronic wounds were more likely to be infected with $P a$ strains producing Pf phage. At the end of the study period, all wounds were classified into groups based on the age of the wound, with cutoffs set at $\leq 3$ months, $\leq 6$ 
months, $\leq 9$ months, $\leq 12$ months, or $>12$ months. They were then further classified as $\operatorname{Pf}(+)$ vs $\operatorname{Pf}(-)$. We found that $0 \%(0 / 3)$ of wounds $\leq 3$ months were $\mathrm{Pf}(+) ; 25 \%(2 / 8)$ wounds $\leq 6$ months of age were $\operatorname{Pf}(+)$; $41.7 \%(5 / 12)$ of wounds $\leq 9$ months of age were $\operatorname{Pf}(+) ; 50 \%(7 / 14)$ of wounds $\leq 12$ months of age were $\operatorname{Pf}(+)$; and $81.8 \%(18 / 22)$ of wounds $>12$ months of age were $\operatorname{Pf}(+)(n=36, p=0.007$ by Chi-square test $)$

(Figure 5D). Wounds infected with $\operatorname{Pf}(+)$ strains of $P a$ were significantly older than $\operatorname{Pf}(-)$ strains (mean age 3.8 years vs. 2.0 years, respectively; median age 2.1 years vs 0.5 years, respectively; $p=0.046$ by Mann Whitney test) (Figure 5E). Moreover, a survival analysis using the endpoint of wound closure showed a significant difference between time to wound closure of the $\operatorname{Pf}(+)$ wounds vs $\operatorname{Pf}(-)$ wounds $(p=0.013$ by Gehan-Breslow-Wilcoxon test) (Figure 5F). Together, these data demonstrate that Pf phage production is associated with clinically-defined chronic wounds.

\section{Pf phage are associated with wound progression}

Given the association between the presence of Pf phage and chronic / non-healing wounds, we next asked whether patients infected with $\operatorname{Pf}(+) P a$ strains indeed have impaired wound healing compared to patients infected with Pf(-) $P a$ strains. To this end, we measured changes in wound dimensions over time in the $36 \mathrm{~Pa}$-positive patients, starting with measurements taken at the patient's initial clinic visit. We found that $\operatorname{Pf}(+)$ patients were more likely to experience an increase in wound size over time than patients who were Pf(-) (8/25 versus 0/11, respectively; $p=0.033$ by two-sided Chi square test) (Figure 5G-H). These data indicate that $\operatorname{Pf}(+)$ strains of $P a$ are associated with delayed wound healing. 


\section{$\underline{\text { Discussion }}$}

We report that Pf phage promote chronic wounds and impaired healing in mice. Pf phage delayed healing in these animals in both the absence or presence of live $P a$, indicative of effects on wound healing independent of effects on chronic infection. Further, in a prospective cohort study in humans, Pf phage were associated with more chronic wound infections and wound size progression. Together, these data strongly implicate Pf phage in chronic wounds and delayed wound healing.

These effects of Pf phage on delayed healing were associated with impaired re-epithelialization. Re-epithelialization is critical for wound closure and its failure leads wounds open to reinjury, re-infection, and perpetuation of a chronic wound (Barrientos et al., 2008; Pastar et al., 2014; Sivamani et al., 2007). Our data are consistent with reports that $P a$ infections can cause impaired epithelialization (Roy et al., 2014) although this was not previously attributed to Pf phage.

Our data implicate crosstalk between immune cells and keratinocytes in these effects. Conditioned media from Pf-treated monocytes prevented keratinocyte migration in vitro. However, direct treatment of keratinocytes with Pf did not, indicating that Pf effects on re-epithelialization are indirect. We previously reported that Pf phage impact production of multiple cytokines and chemokines (Secor et al., 2017; Sweere et al., 2019b; Van Belleghem et al., 2017; Van Belleghem et al., 2018). We have identified multiple factors in this study as well, including several known to have effects on keratinocyte migration and function (Balaji et al., 2015; Banno et al., 2004). In addition to chemokines, other soluble factors present in the wound environment also impact wound healing and might also contribute to the effects reported here (Tu et al., 2019; Williams et al., 2018). Together, these data point to Pf internalization by immune cells present in the wound bed, alternations in the inflammatory milieu, and indirect effects on keratinocyte migration leading to delayed re-epithelialization. However, we cannot exclude potential roles for other cell types within the wound bed.

These data are consistent with other reports implicating Pf phage in the pathogenesis of $\mathrm{Pa}$ infections. Pf contributes to $P a$ virulence in lung infections in mice (Rice et al., 2009; Secor et al., 2015b) and in humans (Burgener et al., 2019). We ourselves previously reported that Pf phage contribute to biofilm 
formation (Secor et al., 2015a; Secor et al., 2015b) and promote bacterial phenotypes associated with chronic infections (Secor et al., 2017). This work also builds on our recent report that Pf phage promote the establishment of wound infections (Sweere et al., 2019b). However, while that study focused on bacterial clearance and initial wound infections, the present work focuses on re-epithelialization and wound resolution.

It is notable that the incidence of $\mathrm{Pa}$ in our patient sample (35\%) is higher than what is typically reported in other cohorts (Panagea and Galloway, 1998). However, because our patients represent a random cross section of individuals referred to the Infectious Disease service, they are likely to include sicker, more complicated patients than might be seen in a general medicine service. It will be important to assess the prevalence of Pf phage in $\mathrm{Pa}$ infections in other contexts and at other sites.

A restriction of our study is its limited prospective cohort design, which does not permit us to follow all patients, especially those with more chronic wounds, for longer than two years. Larger longitudinal studies will allow us to confirm our findings. Since we did not find differences in antibiotic resistance or co-infection profiles between $\mathrm{Pf}(+)$ and $\mathrm{Pf}(-) P a$-infected wounds, we suspect that these factors do not serve as confounding variables. Lastly, chronic wound infection is a complex disease characterized by multiple factors that contribute to disease severity and progression, which can only be controlled for through longitudinal studies with larger samples. Our ongoing work aims to further investigate Pf phage pathogenicity though a larger prospective longitudinal study.

We conclude that Pf phage may provide a novel biomarker and therapeutic target for the delayed wound healing that occurs in the context of $P a$ wound infections. This finding may have particular relevance given our recent report that a vaccine targeting Pf could prevent initial $\mathrm{Pa}$ wound infection (Sweere et al., 2019b). Future longitudinal studies in independent cohorts are needed to validate these studies. 


\section{Acknowledgements}

M.S.B. was supported by the Stanford Bio-X Fellowship. C.D.V. was supported by grant T32 AI007502-

22. J.M.S. was supported by the Gabilan Stanford Graduate Fellowship for Science and Engineering and the Lubert Stryer Bio-X Stanford Interdisciplinary Graduate Fellowship. P.L.B. was supported by grants R21AI133370, R21AI133240, R01AI12492093, and grants from Stanford SPARK, the Falk Medical Research Trust, and the Cystic Fibrosis Foundation (CFF).

\section{$\underline{\text { Author Contributions }}$}

M.S.B. contributed to the study design, conducted experiments, acquired and analyzed data, and prepared the manuscript. C.D.V. provided methods, conducted experiments, analyzed data, and revised the manuscript. J.M.S. provided methods and assisted in conducting experiments, data analysis, and manuscript revisions. E.B.B. provided methods and assisted in data analysis. S.B. and S.K. provided methods and assisted in conducting experiments and data analysis. M.B. and V.S. assisted in conducting experiments and data analysis. P.L.B. and G.A.S. provided oversight in study design, conducting experiments, data analysis, and manuscript revision.

\section{$\underline{\text { Declaration of Interests }}$}

The authors declare no competing interests. 


\section{Figures and Tables}

A.

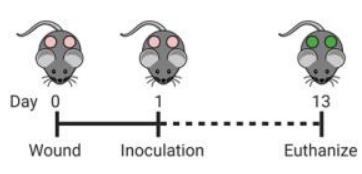

B.

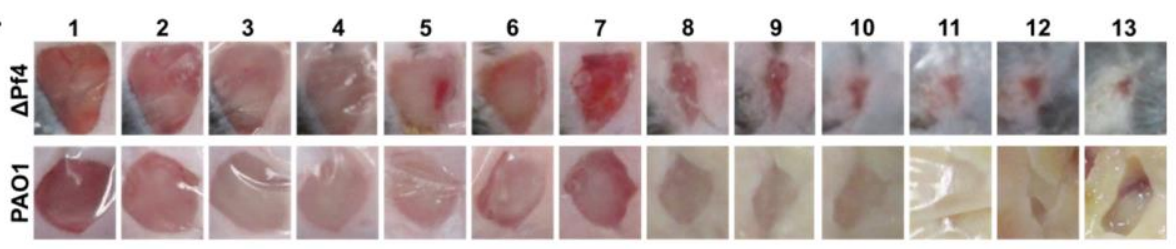

D.

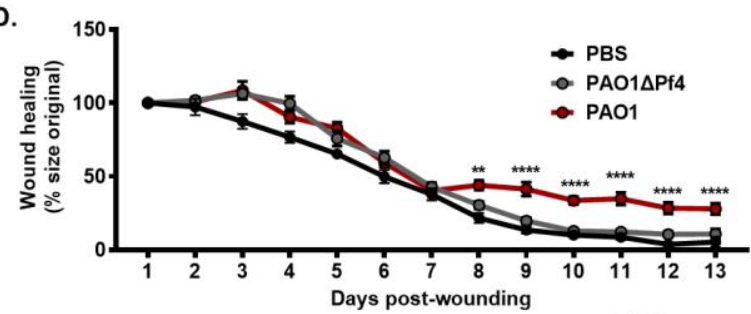

C.

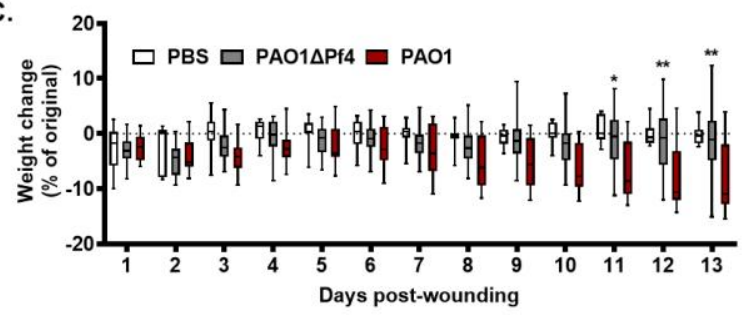

E.

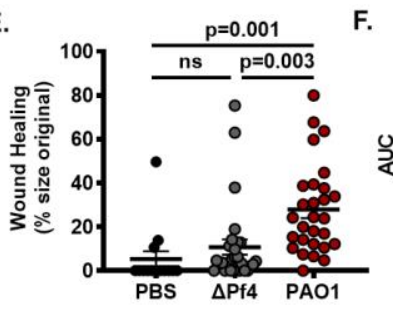

F.

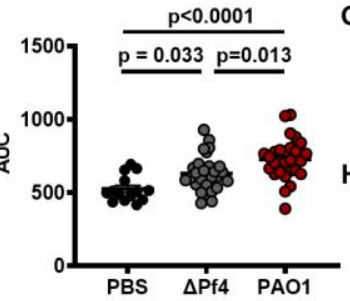

G.

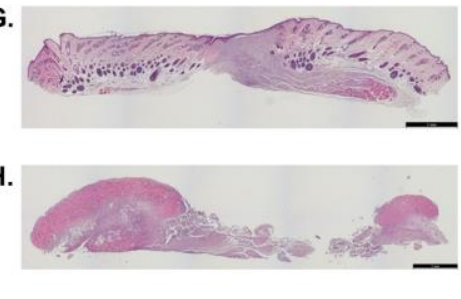

I.

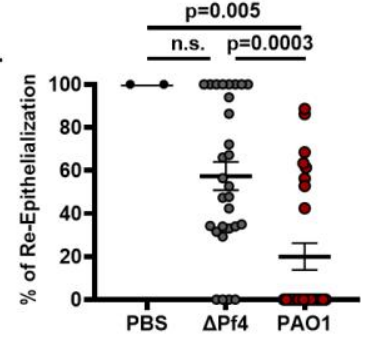

Figure 1. Pf phage lead to decreased wound healing in murine models.

A) Schematic of the full thickness delayed-inoculation chronic $P a$ wound infection murine model. B) Images of representative mice from PAO $\triangle \mathrm{Pf} 4$ and PAO1groups from Day 1-13 post-wounding. C) Weight loss of mice across all days post-wounding (For PAO1 vs PAO1 $\Delta$ Pf4, Day 11: $\mathrm{p}<0.05$; Day 12: $\mathrm{p}<0.01$; Day 13: $\mathrm{p}<0.01$; two-way ANOVA). D) Wound healing rates for all wounds across all days from Day 1-13 post wounding. $\mathrm{n}=28$ wounds in PAO $\triangle \mathrm{Pf} 4$ and PAO1groups; $\mathrm{n}=14$ wounds in PBS group. Results are mean $\%$ size of original wound \pm SE. $* * p<0.005 * * * * p<0.0001$ by one-way ANOVA E) Wound healing at Day 13 post-wounding measured as percentage of original wound area. F) Area-under-curve (AUC) analysis for wound healing rates compiled for all wounds for all Days 1-13 post-wounding ( $\mathrm{n}=28$ wounds in PAO $\triangle \mathrm{Pf} 4$ and PAO1groups; $\mathrm{n}=14$ wounds in PBS group; comparison by one-way ANOVA). G) Representative H\&E stain of Day 13 wound infected with PAO1 $\triangle \mathrm{Pf} 4 . \mathrm{H})$ Representative H\&E stain of Day 13 wound infected with PAO1. I) Percent re-epithelialization calculated as ((Day 1 epithelial gap - Day 13 epithelial gap)/Day 1 epithelial gap). ( $\mathrm{p}=0.005 ; \mathrm{p}=0.0003$ by one-way ANOVA). 

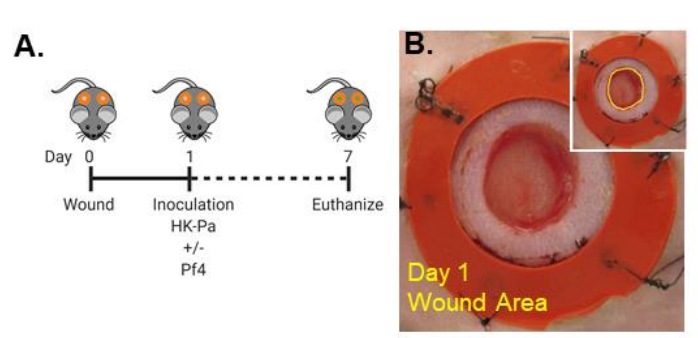
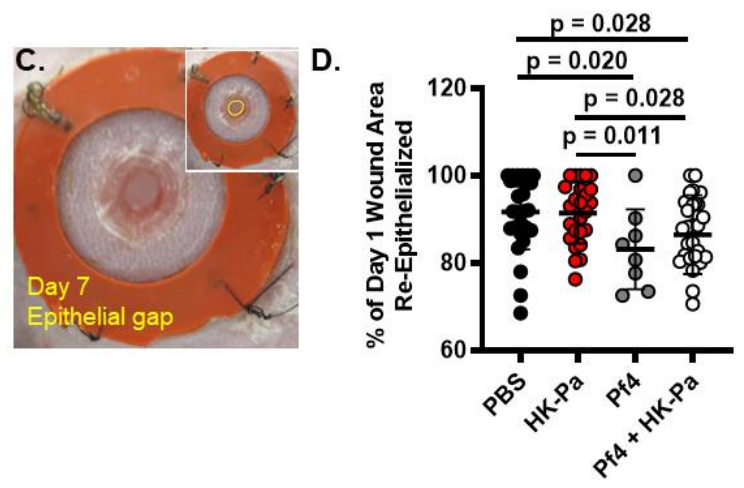

Figure 2. Pf phage delay re-epithelialization in the absence of bacterial infection in murine models. A) Schematic of full-thickness chronic $P a$ wound infection murine model using ring system. B) Image of a representative wound area inoculated with heat-killed $P a$ on Day 1. C) Image of the same wound area inoculated with heat-killed $P a$ on Day 7. D) Epithelial gap on Day 7 normalized to Day 1 wound area. Combined results from multiple experiments with 8-30 wounds in total per group. PBS vs Pf4 + HK-Pa $\mathrm{p}=0.028$, PBS vs Pf4 p=0.020, HK-Pa vs Pf4 p=0.011, HK-Pa vs Pf4 + HK-Pa p=0.028 by unpaired Student's T-test. 
A.

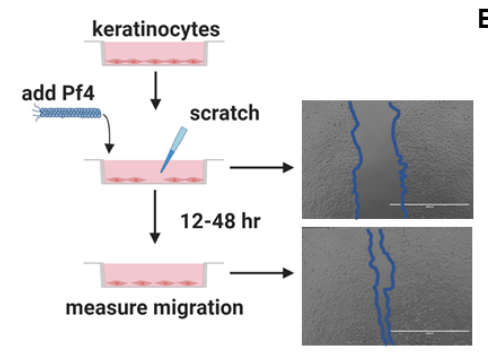

D.

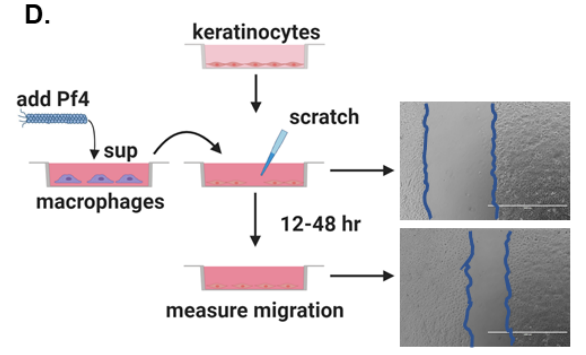

B.

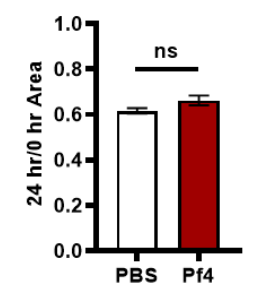

E.

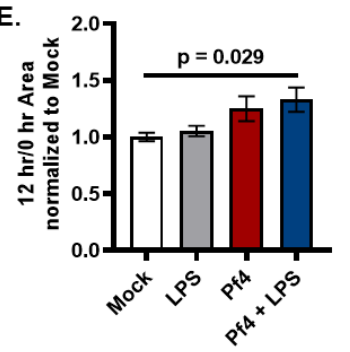

C.

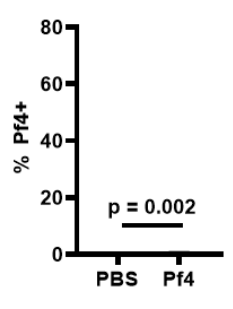

F.

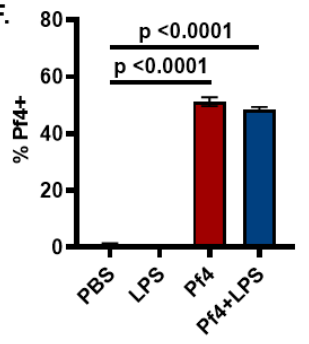

Figure 3. Supernatant from macrophages stimulated with Pf phage slows keratinocyte migration.

A) Schematic of fibroblast and keratinocyte migration assays with representative images of migration assay of HaCaT keratinocytes at 0 hour and 24 hours. B) Effect of Pf4 $\left(1 \times 10^{10}\right.$ copy \#/ml $)$ on HaCaT keratinocyte migration, presented as area at 24 hours/area at 0 hours. Results representative of 3 independent experiments. C) Percentage of Pf4 phage uptake of HaCaT keratinocytes after culture for 3 hours with $1 \times 10^{11}$ copy \#/ml AlexaFluor 488 labeled Pf4 phage. Combined data from 2 independent experiments. D) Schematic of keratinocyte migration assays in which $\mathrm{HaCaT}$ keratinocytes were incubated with supernatant from U937-derived macrophages primed with Pf4 and stimulated with LPS. E) Effect on keratinocyte migration of supernatant from U937-derived macrophages that had been primed with Pf4 $(1 \times$ $10^{10}$ copy \#/ml) overnight and stimulated LPS $(1 \mathrm{ug} / \mathrm{ml})$ for 4 hours. Results are combined from 6 independent experiments presented as area at 12 hours/area at 0 hours normalized to the mock phage prep condition. F) Percentage of Pf4 phage uptake by U937-derived macrophages primed with Pf4 $\left(1 \times 10^{10}\right.$ copy \#/ml) overnight and stimulated LPS $(1 \mathrm{ug} / \mathrm{ml})$ for 4 hours. $\mathrm{P}<0.0001$ by one-way ANOVA. Representative of 2 independent experiments. 

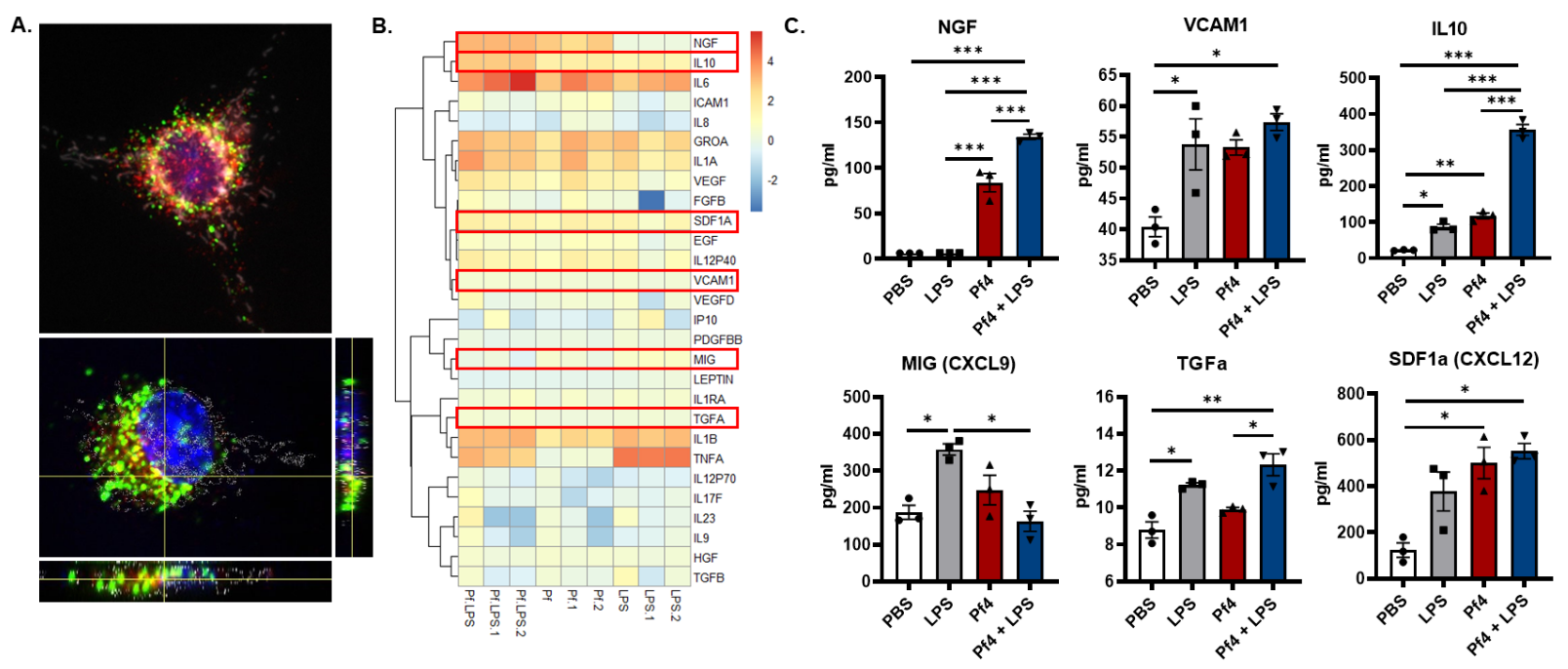

Figure 4. Pf phage uptake by macrophages alters cytokines that affect keratinocyte migration

A) Confocal imaging of murine bone marrow derived macrophage (BMDMs) showing internalization of AlexaFluor 488 labeled Pf4 phage. B) Heat map from Luminex Immunoassay performed on supernatant from human primary monocytes stimulated with Pf4 and LPS. Results are presented as log 2 fold change of concentration compared to PBS control. C) Interpolated values $(\mathrm{pg} / \mathrm{ml})$ of select cytokines and growth factors from Luminex assay of human primary monocytes stimulated with Pf4 and LPS. *p<0.05, $* * \mathrm{p}<0.01, * * * \mathrm{p}<0.001$ by one-way ANOVA. 

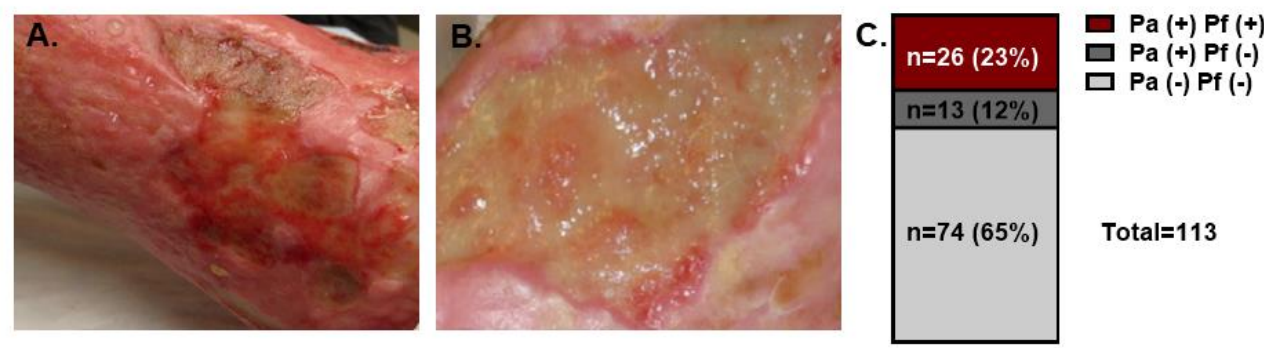

D.

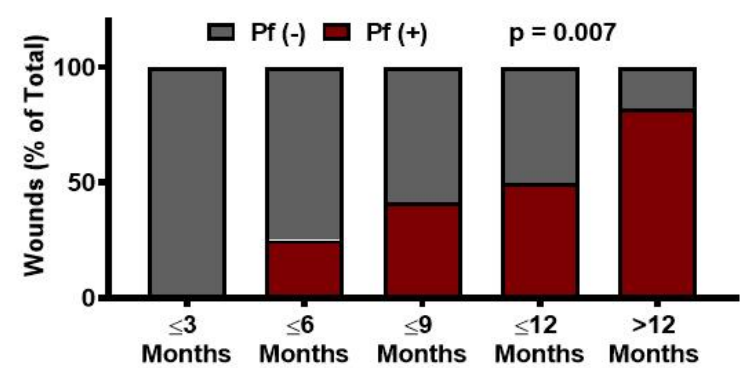

E.

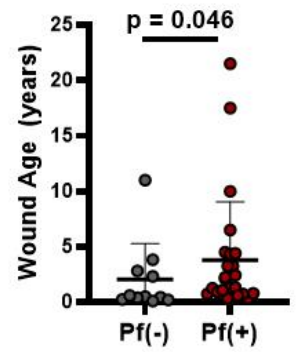

F.

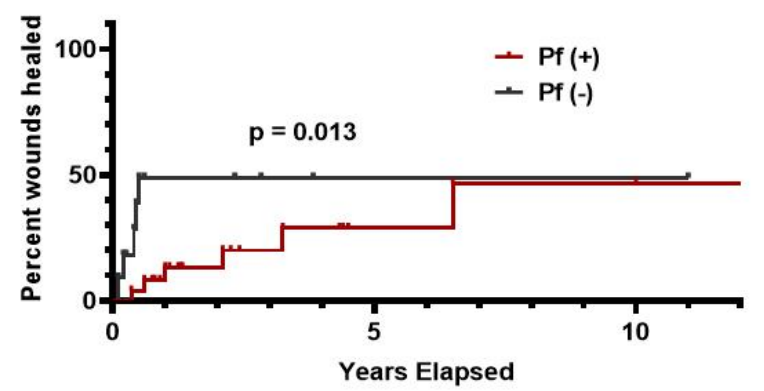

G.
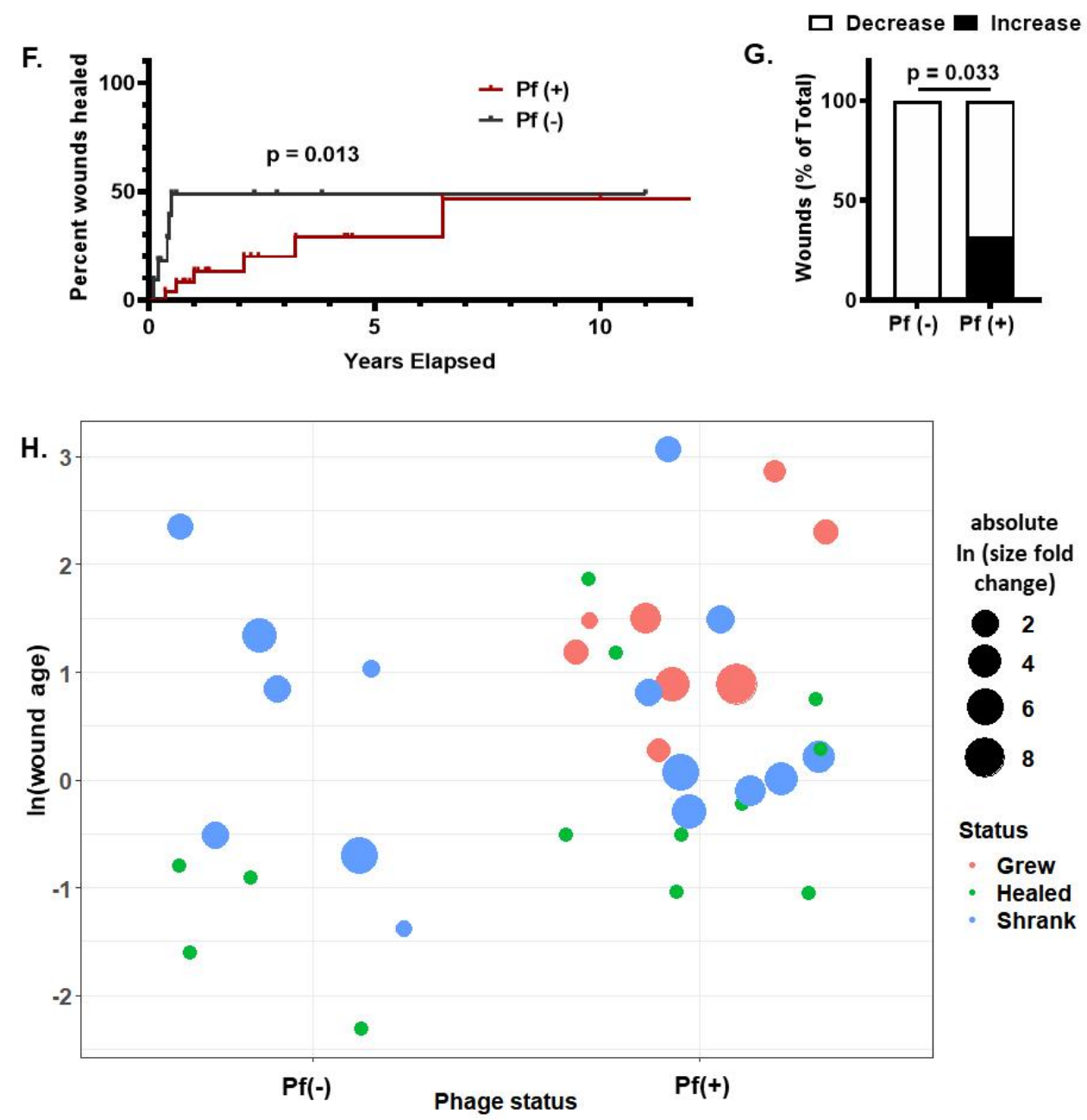

absolute In (size fold change)

2

4

6

C 8

Status

- Grew

- Healed

- Shrank 
Figure 5. Pf phage are associated with more chronic wound infections and increased wound size in humans.

A) Representative image of $\operatorname{Pf}(+)$ wound. B) Representative image of $\operatorname{Pf}(-)$ wound. C) $P a$ and Pf phage prevalence in a cohort of 113 patients seen at the Stanford Advanced Wound Care Center. D) Prevalence of $\operatorname{Pf}(+)$ wounds when grouped by age of wound into $\leq 3$ months, $\leq 6$ months, $\leq 9$ months, $\leq 12$ months, or $>12$ months. Results presented as $\%$ of total wounds in each age group. $n=36 ; p=0.007$ by Chi-square test. E) $\operatorname{Pf}(-)(\mathrm{n}=11)$ and $\mathrm{Pf}(+)(\mathrm{n}=25)$ wound age presented in years. Mean wound age $\mathrm{Pf}(-) 2.0$ years vs $\operatorname{Pf}(+) 3.8$ years. Median wound age $\operatorname{Pf}(-) 0.5$ years vs $\operatorname{Pf}(+) 2.1$ years, $\mathrm{p}=0.046$ by Mann-Whitney test. F) Survival analysis of $\operatorname{Pf}(+)$ and $\operatorname{Pf}(-) P a$ wound infections, with wound healing as end point. Wounds that were not healed by the end of the study were censored. $\mathrm{p}=0.013$ by Gehan-Breslow-Wilcoxon test. $\mathrm{G})$ $\operatorname{Pf}(+)$ status is associated with increases in wound size. $\operatorname{Pf}(-): n=11 ; \operatorname{Pf}(+): n=25 ; p=0.033$ by Chi square test. I) Plot showing wound age and change in size. Wounds were plotted based on $\operatorname{Pf}(-)$ or $\operatorname{Pf}(+)$ phage status and wound age (calculated as $\ln$ (wound age in years)). Wound size change is represented as absolute ln fold change, with color indicating whether wound grew (red), healed (green), or shrank (blue). 
Table 1. Patient Demographic Data.

\begin{tabular}{|c|c|c|c|}
\hline & $\mathrm{Pa}(+) \operatorname{Pf}(+)$ & $\mathrm{Pa}(+) \operatorname{Pf}(-)$ & p-value \\
\hline Samples (n, \%) & $25,69 \%$ & $11,31 \%$ & - \\
\hline $\begin{array}{l}\text { Age of Patients (years) } \\
\text { Mean (SD) }\end{array}$ & $61.6(14.7)$ & $76.6(14.5)$ & $\underline{0.008}$ \\
\hline $\begin{array}{l}\text { Gender }(n) \\
\text { Male } \\
\text { Female }\end{array}$ & $\begin{array}{c}9(36 \%) \\
16(64 \%)\end{array}$ & $\begin{array}{l}6(55 \%) \\
6(45 \%)\end{array}$ & 0.465 \\
\hline $\begin{array}{l}\text { Race/Ethnicity } \\
\text { Caucasian } \\
\text { Asian } \\
\text { Hispanic } \\
\text { African-American }\end{array}$ & $\begin{array}{c}17(68 \%) \\
3(12 \%) \\
3(12 \%) \\
2(8 \%)\end{array}$ & $\begin{array}{c}5(45 \%) \\
1(9 \%) \\
5(45 \%) \\
0(0 \%)\end{array}$ & 0.144 \\
\hline $\begin{array}{l}\text { Body Mass Index }\left(\mathrm{kg} / \mathrm{m}^{2}\right) \\
\quad \text { Mean (SD) }\end{array}$ & 27.95 (11.9) & $30.28(6.9)$ & 0.551 \\
\hline $\begin{array}{l}\text { Age of Wound (years) } \\
\text { Median }\end{array}$ & 2.1 & 0.5 & $\underline{0.046}$ \\
\hline Recurrence of Infection & $9(43 \%)$ & $3(43 \%)$ & 0.756 \\
\hline $\begin{array}{l}\text { Co-Morbidities } \\
\text { Diabetes Mellitus } \\
\text { Renal Disease }\end{array}$ & $\begin{array}{l}12(48 \%) \\
8(32 \%)\end{array}$ & $\begin{array}{c}3(27 \%) \\
1(9 \%)\end{array}$ & $\begin{array}{l}0.295 \\
0.223\end{array}$ \\
\hline
\end{tabular}

Clinical information on patients from the AWCC with culture-positive and qPCR-positive $P a$-infected non-healing wounds who participated in the wound swab study. Renal Disease was defined as patients with Chronic Kidney Disease (CKD) or End Stage Renal Disease (ESRD). Statistical significance was measured using Fisher's Exact Test for the following parameters: Gender, Infection Recurrence, and CoMorbidities. Statistical significance was measured using an Unpaired T-Test for Age and BMI, ChiSquare Test for Race/Ethnicity, and Unpaired Two-Tailed Mann-Whitney Test for Age of Wound because the data was nonparametric. 9 patients were excluded from the Recurrence of Infection analysis because they did not provide an answer on the patient intake form provided by the AWCC. 
Table 2. Human Wound Microbiology Data.

\begin{tabular}{|c|c|c|c|}
\hline & $\mathrm{Pa}(+) \mathrm{Pf}(+)$ & $\mathrm{Pa}(+) \operatorname{Pf}(-)$ & p-value \\
\hline $\begin{array}{l}\text { Pf Phage, copies/swab, mean } \\
\text { (range) }\end{array}$ & $\begin{array}{l}2.16 \times 10^{7} \\
\left(3.55 \times 10^{3}-2.69 \times 10^{8}\right)\end{array}$ & 0 & - \\
\hline $\begin{array}{l}\text { P. aeruginosa, copies/swab, mean } \\
\text { (range) }\end{array}$ & $\begin{array}{l}7.55 \times 10^{7} \\
\left(6.82 \times 10^{2}-9.2 \times 10^{8}\right)\end{array}$ & $\begin{array}{l}2.74 \times 10^{7} \\
\left(5.56 \times 10^{3}-1.79 \times 10^{8}\right)\end{array}$ & 0.395 \\
\hline \multicolumn{4}{|l|}{ Antimicrobials at Time of Swab } \\
\hline Antimicrobials & $14(56 \%)$ & $2(18 \%)$ & 0.067 \\
\hline Anti-Pseudomonal & $6(24 \%)$ & $2(18 \%)$ & $>0.999$ \\
\hline \multicolumn{4}{|l|}{ Antibiotic Resistance } \\
\hline Levofloxacin & $8(32 \%)$ & $1(9 \%)$ & 0.223 \\
\hline Ciprofloxacin & $5(20 \%)$ & $1(9 \%)$ & 0.643 \\
\hline Imipenem & $4(16 \%)$ & $4(36 \%)$ & 0.214 \\
\hline Meropenem & $4(16 \%)$ & $3(27 \%)$ & 0.650 \\
\hline Gentamicin & $2(8 \%)$ & $1(9 \%)$ & $>0.999$ \\
\hline Piperacillin & $1(4 \%)$ & $1(9 \%)$ & 0.524 \\
\hline \multicolumn{4}{|l|}{ Co-Infection } \\
\hline Presence of Co-Infection & $19(76 \%)$ & $7(64 \%)$ & 0.454 \\
\hline Staphylococcus aureus & $10(40 \%)$ & $4(36 \%)$ & $>0.999$ \\
\hline Gram-Positive & $8(32 \%)$ & $2(18 \%)$ & 0.690 \\
\hline Other Gram-Negative & $11(44 \%)$ & $5(45 \%)$ & $>0.999$ \\
\hline Candida Species & $0(0 \%)$ & $1(9 \%)$ & 0.306 \\
\hline
\end{tabular}

Statistical significance for $P a$ copies/swab was analyzed using an Unpaired T-Test. Statistical significance for Antimicrobials at Time of Swab, Antibiotic Resistance, and Co-Infection was analyzed 
bioRxiv preprint doi: https://doi.org/10.1101/2020.03.10.985663; this version posted March 11, 2020. The copyright holder for this preprint (which was not certified by peer review) is the author/funder. All rights reserved. No reuse allowed without permission.

using Fisher's Exact Test. Anti-Pseudomonal antibiotics included in the analysis were ciprofloxacin and levofloxacin. 0 patients were resistant to Tobramycin. None of the Co-Infection parameters were significant, which strengthens the correlation between Pf phage and clinical outcomes. 


\section{Methods}

\section{LEAD CONTACT AND MATERIALS AVAILABILITY}

- Further information and requests for resources and reagents should be directed to and will be fulfilled by the Lead Contact, Paul L. Bollyky (pbollyky@ stanford.edu).

- This study did not generate new unique agents.

\section{EXPERIMENTAL MODEL AND SUBJECT DETAILS}

\section{Bacterial strains and culture conditions}

P. aeruginosa strain PAO1 was used for all experiments. Isogenic phage-free strain PAO1 $\triangle \mathrm{Pf} 4$ is derived from strain PAO1, but PAO1 $\triangle$ Pf4 lacks the genomic copy of Pf4 phage entirely (Rice et al., 2009). This strain can still be re-infected by Pf4 phage. In general, bacteria were prepared as follows. Frozen glycerol stocks were streaked on Luria-Bertani (LB) agar containing selective antibiotics (PAO1 and clinical strains: none; PAO1 $\Delta \mathrm{Pf} 4: 10 \mu \mathrm{g} / \mathrm{mL}$ gentamicin) and grown overnight at $37^{\circ} \mathrm{C}$. An isolated colony was picked and grown overnight at $37^{\circ} \mathrm{C}$ in $\mathrm{LB}$ medium, $\mathrm{pH} 7.4$ under shaking, aerobic conditions. The next day, cultures were diluted to OD600 $=0.05$ in $75 \mathrm{~mL} \mathrm{LB}$ media and cultures were grown until early exponential phase (OD600 \pm 0.3$)$. OD600 was measured and the required number of bacteria was calculated, washed, and prepared as described in the experiments.

\section{Cell Culture Conditions}

Human primary monocytes, U937 cell line (ATCC CRL-1593.2), and other immune cells were cultured in RPMI with 10\% FBS, $100 \mathrm{IU}$ penicillin, $100 \mu \mathrm{g} / \mathrm{mL}$ streptomycin and $1 \mathrm{mM}$ sodium pyruvate. HaCaT human keratinocyte cell line (AddexBio, Cat. No. T0020001) was cultured in DMEM with 10\% FBS, 100 IU penicillin, and $100 \mathrm{ug} / \mathrm{mL}$ streptomycin in T-75 flasks (Corning, Cat. No. 430641). All cells were cultured on sterile tissue culture- treated plates (Falcon). Cells were cultured at $37^{\circ} \mathrm{C}, 5 \% \mathrm{CO} 2$ at a $90 \%$ humidified atmosphere. 


\section{Mice}

Mice were bred and maintained under specific pathogen-free conditions, with free access to food and water, in the vivarium at Stanford University. Mice that underwent surgery received additional Supplical Pet Gel (Henry Schein Animal Health, Cat. No. 029908) and intraperitoneal injections of sterile saline (Hospira, Cat. No. 0409-4888-10). All mice used for in vivo infection experiments were littermates. Conventional C57BL/6J mice were purchased from The Jackson Laboratory (Bar Harbor, ME). All experiments and animal use procedures were approved by the Institutional Animal Care \& Use Committee at the School of Medicine at Stanford University.

\section{Collection of wound swabs from human patients}

From 06/2016 to 06/2018, patients visiting the Stanford Advanced Wound Care Center (AWCC) in Redwood City, California with open wounds were swabbed in duplicate over a one square inch area using Levine's technique, using nylon-flocked wet swabs (Copan Diagnostics, Cat. No. 23-600- 963). Swabs were collected in PBS and stored in $-80^{\circ} \mathrm{C}$ before transport on dry ice. In the laboratory, the swabs in PBS were thawed, vortexed vigorously for 15 seconds, and the contents were aliquoted for quantitation of $P a$ rpIU gene and Pf prophage gene PAO717, as detailed below. Patients at the Wound Care Center were also swabbed for confirmation by diagnostic laboratory culture for the presence of $P a$. Patients were subsequently followed until wounds completely healed or until August 2018. Patients were considered $P a$ positive if their swabs had detectable $P a$ rpIU and their diagnostic cultures were positive. Patients were considered Pf phage-positive if both duplicate wound swabs had detectable levels of Pf phage genes. None of the $P a$-negative patients had detectable Pf phage. For patients with multiple wounds, the dominant wound was selected for analysis. Patient enrollment and swab collection were done in compliance with the Stanford University Institutional Review Board for Human Research. Written informed consent was obtained from each patient before swab collection. 


\section{Human wound closure analysis}

Length, width, and depth measurements of the wounds were taken and recorded into the patient's flowsheet at the start of each visit by the intake nurse. Additional measurements of undermining and tunneling were also taken when applicable. Wound measurements were taken for all patients in the study each time they visited the Stanford Advanced Wound Care Center (AWCC). The AWCC nursing staff is trained in collecting and recording the longest length, width, and depth for each wound. Total volume was calculated for each wound for analysis. Wound healing was defined as a reduction in size compared to the first recorded measurement, whereas wound size increase was defined as an increase in wound volume compared to the first recorded measurement. Wound images were obtained directly from the EMR and uploaded at each clinic visit by the AWCC staff from the designated clinic camera used for this purpose. Images were cropped and resized to protect patient identity. Informed consent was obtained from patients before images were taken. Patient data was collected from EMR chart records, including patient age, gender, co-morbidities, wound age, and other variables. This included history and physicals, progress notes, and documents uploaded into the EMR, such as the AWCC patient intake questionnaire. Patient flowsheet review was accessed for precise wound measurements and laboratory results were accessed to assess renal function and glycemic control. Microbiologic data were reviewed for antibiotic resistance profiles.

\section{METHOD DETAILS}

\section{Materials and Reagents}


The following chemicals, antibiotics, and reagents were used: RPMI (HyClone, Cat. No. SH30027.01); DMEM (Hyclone, Cat. No. SH30243.01); PBS (Corning Cellgro, Cat. No. 21-040-CV); tryptone (Fluka Analytical, Cat. No. T7293); sodium chloride (Acros Organics, Cat. No. 7647-14-5); yeast extract (Boston BioProducts, Cat. No. P-950); agar (Fisher BioReagents, Cat. No. BP9744); gentamicin (Amresco, Cat. No. E737).

\section{Preparation of heat-killed bacteria}

Frozen glycerol stocks were streaked on LB agar as described above. Individual colonies were grown in 5 $\mathrm{mL}$ of $\mathrm{LB}$ broth the next day for 2 hours to approximately $2 \times 10^{8} \mathrm{CFU} / \mathrm{mL}$. The bacterial cultures were centrifuged at $6,000 \times g$ for 5 minutes, and the pellet was washed in $1 \mathrm{~mL}$ of PBS three times. Finally, the pellet was resuspended in $1 \mathrm{~mL}$ of PBS and heated for 30 minutes at $90^{\circ} \mathrm{C}$ under shaking conditions. The preparation was checked for sterility by plating.

\section{Phage Purification}

This was performed as previously reported. In brief, phage were harvested following treatment with 1 $\mu \mathrm{g} / \mathrm{mL}$ DNase I (Roche, Cat. No. 4716728001) and PEG8000-precipitation as described previously (Sweere et al., 2019b). In some experiments, supernatant was treated with $250 \mu \mathrm{g} / \mathrm{mL}$ of RNAse A (Thermo Fisher Scientifc, Cat. No. EN0531) or $85 \mathrm{U} / \mathrm{mL}$ of benzonase (Novagen, Cat No. 70746) for 4 hours at $37^{\circ} \mathrm{C}$ before sterilization. The purified phage pellets were suspended in sterile PBS and dialyzed in 10-kDa molecular weight cut-off tubing (FisherScientific, Cat. No. 88243) against PBS, quantified by qPCR, diluted at least $10,000 \times$ to appropriate concentrations in sterile PBS and filter-sterilized. We sent three different Pf4 preparations diluted in PBS to working concentrations $\left(1 \times 10^{8} \mathrm{Pf} 4 / \mathrm{mL}\right)$ to Nelson Labs (Salt Lake City, UT), where they were tested for endotoxin by Limulus amoebocyte lysate testing. All three preps had endotoxin levels under the test sensitivity level of $0.05 \mathrm{EU} / \mathrm{mL}$.

Phages were purified by PEG precipitation only unless noted otherwise. Bacteria were infected with stocks of Pf4 phage at mid-log phase and cultured in $75 \mathrm{~mL}$ of $\mathrm{LB}$ broth for 48 hours at $37^{\circ} \mathrm{C}$ under 
shaking conditions. Bacteria were removed by centrifugation at $6,000 \times g$ for 5 minutes, and supernatant was treated with $1 \mu \mathrm{g} / \mathrm{mL}$ DNase I (Roche, Cat. No. 4716728001 ) for 2 hours at $37^{\circ} \mathrm{C}$ before sterilization by vacuum filtration through a $0.22 \mu \mathrm{M}$ filter. Pf phage were precipitated from the supernatant by adding $0.5 \mathrm{M} \mathrm{NaCl}$ and 4\% polyethylene glycol (PEG) 8000 (Milipore Sigma, Cat. No. P2139). Phage solutions were incubated overnight at $4^{\circ} \mathrm{C}$. Phage were pelleted by centrifugation at $13,000 \times g$ for 20 minutes, and the pellet suspended in sterile TE buffer $(\mathrm{pH} 8.0)$. The suspension was centrifuged for $15,000 \times g$ for 20 minutes, and the supernatant was subjected to another round of PEG precipitation. The purified phage pellets were suspended in sterile PBS and dialyzed in 10- $\mathrm{kDa}$ molecular weight cut-off tubing (FisherScientific, Cat. No. 88243) against PBS, quantified by qPCR, diluted at least $10,000 \times$ to appropriate concentrations in sterile PBS and filter- sterilized.

For migration experiments with U937 supernatants, phage purification was performed by anion exchange. The phage filtrate was subsequently concentrated to $19 \mathrm{ml}$ using Amicon Ultra $15 \mathrm{ml} 50 \mathrm{kDa}$ Ultracel spin filters (Millipore). The $\mathrm{pH}$ of the phage concentrate was adjusted to $\mathrm{pH} 5.5$ using a $250 \mathrm{mM}$ MES buffer ( $\mathrm{pH}$ 5.5) to a final concentration of $25 \mathrm{mM}$ MES. The phage concentrate was passaged through a Pierce Strong Cation Exchange maxi prep columns (Thermo Fisher) as instructed by the manufacturer. The flow through containing the phage was dialyses overnight against $4125 \mathrm{mM}$ Tris- $\mathrm{HCl}(\mathrm{pH} 8)$. The following day the dialyses phage preparation was further purified through a Pierce Strong Anion Exchange (SAX) maxi prep columns (Thermo Fisher) as instructed by the manufacturer. Briefly, the phage preparation was transferred to the SAX column and centrifuged at $500 \mathrm{~g}$ for $5 \mathrm{~min}$. the flow through was discarded and the column was washed once with $25 \mathrm{mM}$ Tris- $\mathrm{HCl}(\mathrm{pH}$ 8). Following the first wash, the column was washed three additional times using $25 \mathrm{mM}$ Tris- $\mathrm{HCl}(\mathrm{pH}$ 8) supplemented with $0.3 \mathrm{M} \mathrm{NaCl}$. In a final step the phage was eluted from the column in three steps using $10 \mathrm{ml}$ of $25 \mathrm{mM}$ Tris- $\mathrm{HCl}(\mathrm{pH}$ 8) supplemented with $1 \mathrm{M} \mathrm{NaCl}$. The purified phage Pf4 was further concentrated using Amicon Ultra $15 \mathrm{ml}$ $50 \mathrm{kDa}$ Ultracel spin filters (Millipore) to a final volume of $2.5 \mathrm{ml}$. The purified phage Pf4 was desalted in a final step using a PD-10 Desalting Columns with Sephadex G-25 Medium (GE Healthcare) and eluted with PBS. The phage concentration was determined through qPCR and Tris-Tricine protein gel. 


\section{Quantification of Pf phage}

As several factors can produce plaques on bacterial lawns (other species of phage, pyocins, host defensins, etc.), we quantitated Pf phage using a qPCR assay as previously described (Secor et al., 2015b). In brief, to quantitate Pf prophage in human or mouse wound homogenates and purified Pf phage preparations, bacterial cells and debris were removed by centrifugation at $8,000 \times \mathrm{g}$ for $10 \mathrm{~min}$. Supernatants were boiled at $100^{\circ} \mathrm{C}$ for 20 min to denature any phage particles, releasing intact Pf phage DNA. $2 \mu \mathrm{L}$ was used as a template in $20 \mu \mathrm{L}$ qPCR reactions containing $10 \mu \mathrm{L}$ SensiFAST ${ }^{\mathrm{TM}}$ Probe Hi-ROX (Bioline, Cat. No. BIO82020), $200 \mathrm{nM}$ probe, and forward and reverse primers with concentrations depending on the target. Cycling conditions were as follows: $95^{\circ} \mathrm{C} 2 \mathrm{~min},\left(95^{\circ} \mathrm{C} 15 \mathrm{sec} ., 60^{\circ} \mathrm{C} 20 \mathrm{sec}.\right)$ x 40 cycles on a StepOnePlus Real-Time PCR system (Applied Biosystems). For a standard curve, the sequence targeted by the primers and probe were inserted into a pUC57 plasmid (Genewiz) and tenfold serial dilutions of the plasmid were used in the qPCR reactions. For the human wound swabs, the primers and probe were designed to recognize PAO717, a gene conserved across the Pf phage family (F at 600 nM: TTCCCGCGTGGAATGC; R at 400 nM: CGGGAAGACAGCCACCAA; probe: AACGCTGGGTCGAAG); the $P a$ 50S ribosomal gene $r p I U$ was used to confirm $\mathrm{Pa}$ infection (F at $200 \mathrm{nM}$ : CAAGGTCCGCATCATCAAGTT; R at $200 \mathrm{nM}$ : GGCCCTGACGCTTCATGT; probe: CGCCGTCGTAAGC). For the Pf4 phage purifications, the primers and probe were designed to recognize a Pf4 phage-specific intergenic region between PAO728 and PAO729 (F at 500 nM: GGAAGCAGCGCGATGAA; R at $500 \mathrm{nM:} \mathrm{GGAGCCAATCGCAAGCAA;} \mathrm{probe:}$ CAATTGCGCTGGTGAA). For the Pf phage purification preparations, levels of $\mathrm{Pa}$ 50S ribosomal gene rpIU were measured to correct for contaminating genomic $\mathrm{Pa}$ DNA, but those levels were usually negligible.

\section{Fluorescent labeling of Pf phage}

Purified Pf4 preparations were labeled with AlexaFluor 488-labeled TFP ester (Molecular Probes, Cat. No. A37570) following the manufacturer's protocol. Following labeling, labeled Pf4 viral particles were 
separated from unincorporated dye using PD10 Sephadex G-25 desalting columns (GE Healthcare, Cat. No. 17085101) according to manufacturer's instructions. The labeled viral particles were then quantified using qPCR.

\section{Uptake assays of labeled Pf phage by HaCaT keratinocytes}

HaCaT cells were seeded at $1.2 \times 10^{5}$ cells per well in 24 well plates and incubated overnight at $37^{\circ} \mathrm{C}$. AlexaFluor 488-labeled Pf4 phage was added to wells for a final concentration of $1 \times 10^{11}$ labeled Pf4/ml. Cells were incubated for 3 hours at $37^{\circ} \mathrm{C}$ for uptake analysis or $4^{\circ} \mathrm{C}$ for adsorption analysis. Cells were removed from culture plate with $0.25 \%$ trypsin-EDTA solution, washed in cold flow cytometry buffer twice, stained for live-dead discrimination, and fixed according to our flow cytometric protocol. Acquis ion was performed on a LSR II flow cytometer (BD Biosciences) or Aurora spectral flow cytometer (Cytek). Analysis was performed using FlowJo (BD Life Sciences).

\section{In vivo murine full-thickness wound infection model}

This was done as recently described (Sweere et al., 2019a; Sweere et al., 2019b). In brief, ten-to-twelveweek old male mice were anesthetized using 3\% isoflurane, and their backs were shaved using a hair clipper and further depilated using hair removal cream (Nair). The shaved area was cleaned with sterile water and disinfected twice with Betadine (Purdue Fredick Company, Cat. No. 19-065534) and 70\% ethanol. Mice received 0.1-0.5 mg/kg slow-release buprenorphine (Zoopharm Pharmacy) as an analgesic. Mice received two dorsal wounds by using 6-mm biopsy punches to outline the wound area, and the epidermal and dermal layer were excised using scissors. For certain experiments as indicated in the text, a silicone ring was sutured in place around the wound. The wound area was washed with saline and covered with Tegaderm (3M, Cat. No. 1642W). Bacteria were grown as described above and diluted to $1 \times 10^{7} \mathrm{CFU} / \mathrm{mL}$ in PBS. Mice were inoculated with $40 \mu \mathrm{L}$ per wound 24 hours post-wounding, and control mice were inoculated 
with sterile PBS. Mice were weighed daily and given Supplical Pet Gel and intraperitoneal injections of sterile saline. Upon takedown, wound beds were excised and processed for histological analysis.

\section{Wound Healing Analysis in Murine Models}

This was done as previously described (Balaji et al., 2014). In brief, using a dot ruler for standardization, images of both wounds for all mice were taken on each day from Day 1 to Day 13 post-wounding. Images were taken using Canon PowerShot Camera (Item Model No. 1096C001) mounted on a fixed tripod for consistency in the height and angle at which images were taken. Using the images, wound area was measured using Fiji (Image J, National Institutes of Health) (Schindelin et al., 2012) by tracing the border of the wounded area, as well as the border of a standardized dot measure. For histologic analyses, wounds were harvested, bifurcated, fixed in $10 \%$ neutral buffered formalin, and embedded in paraffin. Wound sections of $5 \mu \mathrm{m}$ were cut from paraffin-embedded blocks. Immune cell influx, general tissue morphology, epithelial gap and granulation tissue area were measured from hematoxylin and eosin (H\&E) stained sections using morphometric image analysis (Image-Pro, Media Cybernetics, Silver Spring, MD, and Metamorph, Molecular Devices, Downingtown, PA). Images were obtained using a Nikon Eclipse microscope, and image analysis was performed using the Nikon Elements software (Nikon Instruments, Melville, NY, USA). The percentage of CD45+ cells to the total cell infiltrate was calculated in 6 highpowered fields (HPFs, 64X) for each wound section. The HPFs were chosen just above the panniculus carnosus and were evenly distributed between the two wound epithelial margins. On a $4 \times$ edge-to-edge wound section image, epithelial gap was measured as the distance (in millimeters) between encroaching epithelial margins. The granulation tissue area was measured (in $\mathrm{mm}^{2}$ ) as the entire cellular region within the epithelial margin. Any sample that did not yield reliable counting due to sample quality was excluded from the analysis for that particular variable. All scoring was done by an independent pathologist. 


\section{Microtiter plate crystal violet assay}

Bacterial cells were inoculated in a 96-well polystyrene plate. Plate was incubated at 37C, static condition for 24 and 48 hours. Post incubation, media was discarded and the plate was washed to remove unattached or planktonic bacteria. Wells were stained in crystal violet. Post staining, the plate was washed to remove excessive dye with ethanol and absorbance was measured at $590 \mathrm{~nm}$.

\section{Confocal laser scanning microscopy and Scanning Electron Microscopy of $\mathrm{Pa}$ biofilms}

Bacterial cultures were seeded at $37 \mathrm{C}$ in sterile tryptic soy broth. Polycarbonate membrane (PCM) discs were cut at $1 \mathrm{~cm}$ diameter and placed on tryptic soy agar plates. Overnight broth cultures were spotted at $10 \mu \mathrm{l}$ per disc and culture spots were allowed to dry. Plates were incubated at $37 \mathrm{C}$ for 24,48 , and 72 hours to allow biofilm formation. For the 48 and 72 hour biofilms, the discs were transferred to new tryptic soy discs every 24 hours to replenish nutrients. After respective time intervals, PCM discs were transferred to glass slides. Biofilms were stained with Syto9 (cell stain) and Sypro Ruby (biofilm matrix stain). Post staining, discs were mounted with a coverslip and observed on a confocal laser scanning microscopy at $63 \times$. Thickness measurements were derived from ' $z$ ' values of images. For SEM, biofilms were sputter coated with gold.

\section{Luminex immunoassay}

Human peripheral blood mononuclear cells (PBMCs) were isolated from whole blood using lymphoprep (Stem Cell technologies, Cat no. 07851). Monocytes were subsequently isolated from the PBMC mixture using a human monocyte isolation kit without CD16 depletion (Stem Cell Technologies, Cat no. 19058), as described by the manufacturer. The isolated monocytes were resuspended at $5 \times 10^{7}$ cells $/ \mathrm{ml}$ in RPMI supplemented with MEM (non-essential amino acids, Sodium pyruvate, Penicilline/Streptomycine, Lglutamine $(200 \mathrm{mM}), 2$-mercaptoethanol $(50 \mathrm{mM})$ and $10 \%$ heat inactivated fetal bovine serum. A $100 \mu \mathrm{l}$ of the monocyte suspension was transferred to a 96 well plate and stimulated for $15 \mathrm{~h}$ with $10^{9}$ phage particles. Subsequently LPS $(1 \mu \mathrm{g} / \mathrm{ml})$ was added for $4 \mathrm{~h}$. Supernatant was collected and used for cytokine 
profiling through Luminex (The Human Immune Monitoring Center, Stanford). Human 63-plex kits were purchased from eBiosciences/Affymetrix and used according to the manufacturer's recommendations with modifications as described below. Briefly: Beads were added to a 96 well plate and washed in a Biotek ELx405 washer. Samples were added to the plate containing the mixed antibody-linked beads and incubated at room temperature for 1 hour followed by overnight incubation at $4{ }^{\circ} \mathrm{C}$ with shaking. Cold and Room temperature incubation steps were performed on an orbital shaker at 500-600 rpm. Following the overnight incubation plates were washed in a Biotek ELx405 washer and then biotinylated detection antibody added for 75 minutes at room temperature with shaking. Plate was washed as above and streptavidin-PE was added. After incubation for 30 minutes at room temperature wash was performed as above and reading buffer was added to the wells. Each sample was measured in duplicate. Plates were read using a Luminex 200 instrument with a lower bound of 50 beads per sample per cytokine. Custom assay Control beads by Radix Biosolutions are added to all wells.

\section{Migration Assay}

For HaCaT cell migration assays a standard scratch assay was used (Cory, 2011). In brief, cells were seeded at a density of $1.5 \times 10^{5}$ cells per well in DMEM 10\% FBS in collagen-coated 24-well tissue culture plates. Media was removed and $\mathrm{HaCaT}$ cells were then serum starved in DMEM containing only 2\% FBS for 24 hours prior to the treatment. A scratch defect was created in the cell monolayer along the diameter in each group using a sterile pipette tip (200 $\mu \mathrm{l}$ tip). Treatment groups had Pf4 bacteriophage added to media with final concentration of $1 \times 10^{10} \mathrm{copy} \# / \mathrm{ml}$. Photographic images were obtained at 0 and 24 hours incubation using an Invitrogen EVOS FL Imaging System. The unfilled scratch defect area was measured at each reference point per well. Data was presented as extent of wound closure, that is, the percentage by which the scratch area has decreased at a given time point for each treatment as compared to the original defect (at 0 hours). All experiments were carried out at minimum in triplicate and the passage number was similar amongst the different groups. For experiments utilizing supernatant from U937 macrophages, media was replaced after serum starvation with supernatant prepared as follows. U937 cells were differentiated into 
macrophages by stimulation for 3 days with $100 \mathrm{n} \mathrm{g/ml} \mathrm{Phorbol} \mathrm{12-myristate} \mathrm{13-acetate} \mathrm{(Sigma,} \mathrm{Cat.} \mathrm{No.}$ P1585). Resulting macrophages were seeded in 6-well plates at $2 \times 10^{6}$ cells/well and stimulated with $1 \times$ $10^{10} \mathrm{\#} / \mathrm{ml}$ Pf4 overnight followed by LPS $1 \mathrm{ug} / \mathrm{ml}$ for 4 hours at $37^{\circ} \mathrm{C}$. Supernatant was then centrifuged to remove debris and cells and frozen at $-80^{\circ} \mathrm{C}$.

\section{Generation of murine bone marrow-derived macrophages (BMDMs)}

Femurs were isolated from male or female mice of $>10$ weeks of age and bone marrow was flushed out using a 27 G PrecisionGlide needle (BD Biosciences, Cat. No. 305109). Cells were plated at $1 \times 10^{6}$ cells in $10 \mathrm{ml}$ of media per Petri Dish (Fisherbrand, Cat. No. FB0875711). Media was supplemented with 20 ng/ml of M-CSF (Invitrogen, Cat. No. 14-8983-62). An equivalent amount of fresh media containing cytokines was added three days after plating, and 50\% of media was changed 5 and 7 days after plating. BMDMs were harvested on day 6 after plating. Supernatant containing non-adherent cells was collected, and adherent cells were lifted by the addition of 10\% Accumax in Accutase for $5 \mathrm{~min}$. Both cell populations were pooled to obtain a mixture of mature and immature cells, washed in fresh media at $300 \mathrm{~g}$ for $5 \mathrm{~min}$, and prepared for experiments.

\section{Confocal microscopy}

BMDMs were seeded on glass 12-mm \#1.5H precision coverslips (Thorlabs, Cat. No. CG15CH) coated with poly-L-lysine (Sigma, Cat. No. P8920) or 0.2\% poly-L-ornithine (Sigma, Cat. No. P3655) according to the manufacturer's instructions at a density of $1 \times 10^{6}$ cells/well in a 6-well plate. Cells were either stimulated with coated $1 \times 10^{9} \mathrm{Pf} 4 / \mathrm{ml}$ labeled Pf 4 or soluble $1 \times 10^{10} \mathrm{Pf} 4 / \mathrm{ml}$ labeled Pf 4 for 3 hours. Cells were washed three times with PBS and subsequently fixed with $10 \%$ neutral buffered formalin for 5 minutes at room temperature. After permeabilization with $0.5 \%$ Triton X-100, coverslips were washed twice with PBS and excess formalin was quenched by incubation with $150 \mathrm{mM}$ glycin, pH 8.0 (Fisher BioReagents, Cat. No. BP381) for 30 minutes. Coverslips were blocked with Sea Block blocking buffer (Thermo Scientific, Cat. No. 37527) followed by incubation with primary antibodies in Sea Block overnight at $4^{\circ} \mathrm{C}$. 
Coverslips were washed three times with PBS and incubated with the appropriate fluorescently labeled secondary antibodies. Coverslips were mounted in ProLong Diamond Antifade Mountant (Invitrogen, Cat. No. P36970) with DAPI to label nuclei. Fluorescent specimens were examined using a confocal system (DMX BLAZE 3D SIM, GE Healthcare). Images were acquired using Leica DFC365 FX camera and the Leica Application Suite X (Leica). Image analysis was performed using FIJI (NIH).

\section{QUANTIFICATION AND STATISTICAL ANALYSIS}

All statistical analyses were done using GraphPad Prism (GraphPad Software, Inc. La Jolla, CA). All Unpaired T-Tests, Mann-Whitney Test, Fisher's Exact Tests, and Chi-Square Test were two-tailed. Depicted are Means with Standard Error or Standard Deviation of the population unless otherwise stated. Statistical significance was considered $p<0.05$.

\section{DATA AND CODE AVAILABILITY}

Original/source data for figures and tables in this paper is available by request 


\section{$\underline{\text { References }}$}

Balaji, S., LeSaint, M., Bhattacharya, S.S., Moles, C., Dhamija, Y., Kidd, M., Le, L.D., King, A., Shaaban, A., Crombleholme, T.M., et al. (2014). Adenoviral-mediated gene transfer of insulinlike growth factor 1 enhances wound healing and induces angiogenesis. J Surg Res 190, 367377.

Balaji, S., Watson, C.L., Ranjan, R., King, A., Bollyky, P.L., and Keswani, S.G. (2015). Chemokine Involvement in Fetal and Adult Wound Healing. Adv Wound Care 4, 660-672.

Banno, T., Gazel, A., and Blumenberg, M. (2004). Effects of tumor necrosis factor-alpha (TNF alpha) in epidermal keratinocytes revealed using global transcriptional profiling. J Biol Chem 279, 32633-32642.

Barrientos, S., Stojadinovic, O., Golinko, M.S., Brem, H., and Tomic-Canic, M. (2008). PERSPECTIVE ARTICLE: Growth factors and cytokines in wound healing. Wound Repair Regen 16, 585-601.

Bjarnsholt, T., Kirketerp-Møller, K., Jensen, P.Ø., Madsen, K.G., Phipps, R., Krogfelt, K., Høiby, N., and Givskov, M. (2008). Why chronic wounds will not heal: a novel hypothesis. Wound Repair Regen 16, 2-10.

Bowler, P.G. (2002). Wound pathophysiology, infection and therapeutic options. Ann Med 34, 419-427.

Bryers, J.D. (2008). Medical biofilms. Biotechnol Bioeng 100, 1-18.

Burgener, E.B., Sweere, J.M., Bach, M.S., Secor, P.R., Haddock, N., Jennings, L.K., Marvig, R.L., Johansen, H.K., Rossi, E., Cao, X., et al. (2019). Filamentous bacteriophages are associated with chronic Pseudomonas lung infections and antibiotic resistance in cystic fibrosis. Sci Transl Med 11.

Castang, S., and Dove, S.L. (2012). Basis for the essentiality of H-NS family members in Pseudomonas aeruginosa. J Bacteriol 194, 5101-5109.

Centers for Disease Control and, P. (2013). Antimicrobial Resistance Threats in the United States, 2013, pp. 113.

Clinton, A., and Carter, T. (2015). Chronic Wound Biofilms: Pathogenesis and Potential Therapies. Lab Med 46, 277-284.

Cory, G. (2011). Scratch-wound assay. Methods Mol Biol 769, 25-30.

de Vries, C.R., Sweere, J.M., Ishak, H., Sunkari, V., Bach, M.S., Manasherob, R., and Bollyky, P.L. A Delayed Inoculation Model of Chronic Pseudomonas aeruginosa Wound Infection. JoVE.

Dowd, S.E., Sun, Y., Secor, P.R., Rhoads, D.D., Wolcott, B.M., James, G.A., and Wolcott, R.D. (2008). Survey of bacterial diversity in chronic wounds using pyrosequencing, DGGE, and full ribosome shotgun sequencing. BMC Microbiol 8, 43.

Gardner, S.E., and Frantz, R.A. (2008). Wound bioburden and infection-related complications in diabetic foot ulcers. Biol Res Nurs 10, 44-53. 
Gjødsbøl, K., Christensen, J.J., Karlsmark, T., Jørgensen, B., Klein, B.M., and Krogfelt, K.A. (2006). Multiple bacterial species reside in chronic wounds: a longitudinal study. Int Wound J 3, 225-231.

Gordillo Altamirano, F.L., and Barr, J.J. (2019). Phage Therapy in the Postantibiotic Era. Clin Microbiol Rev 32.

Guo, S., and Dipietro, L.A. (2010). Factors affecting wound healing. J Dent Res 89, 219-229.

Górski, A., Międzybrodzki, R., Węgrzyn, G., Jończyk-Matysiak, E., Borysowski, J., and WeberDąbrowska, B. (2019). Phage therapy: Current status and perspectives. Med Res Rev.

Høiby, N., Ciofu, O., Johansen, H.K., Song, Z.-J., Moser, C., Jensen, P.Ø., Molin, S., Givskov, M., Tolker-Nielsen, T., and Bjarnsholt, T. (2011). The clinical impact of bacterial biofilms. Int J Oral Sci 3, 55-65.

James, G.A., Swogger, E., Wolcott, R., Pulcini, E.D., Secor, P., Sestrich, J., Costerton, J.W., and Stewart, P.S. (2008). Biofilms in chronic wounds. Wound Repair Regen 16, 37-44.

Jesaitis, A.J., Franklin, M.J., Berglund, D., Sasaki, M., Lord, C.I., Bleazard, J.B., Duffy, J.E., Beyenal, H., and Lewandowski, Z. (2003). Compromised host defense on Pseudomonas aeruginosa biofilms: characterization of neutrophil and biofilm interactions. J Immunol 171, 4329-4339.

Järbrink, K., Ni, G., Sönnergren, H., Schmidtchen, A., Pang, C., Bajpai, R., and Car, J. (2016). Prevalence and incidence of chronic wounds and related complications: a protocol for a systematic review. Syst Rev 5, 152.

Kirketerp-Møller, K., Jensen, P.Ø., Fazli, M., Madsen, K.G., Pedersen, J., Moser, C., TolkerNielsen, T., Høiby, N., Givskov, M., and Bjarnsholt, T. (2008). Distribution, organization, and ecology of bacteria in chronic wounds. J Clin Microbiol 46, 2717-2722.

Kirsner, R.S. (2016). The Wound Healing Society chronic wound ulcer healing guidelines update of the 2006 guidelines--blending old with new. Wound Repair Regen 24, 110-111.

Liang, C.-C., Park, A.Y., and Guan, J.-L. (2007). In vitro scratch assay: a convenient and inexpensive method for analysis of cell migration in vitro. Nat Protoc 2, 329-333.

Malic, S., Hill, K.E., Hayes, A., Percival, S.L., Thomas, D.W., and Williams, D.W. (2009). Detection and identification of specific bacteria in wound biofilms using peptide nucleic acid fluorescent in situ hybridization (PNA FISH). Microbiology 155, 2603-2611.

Panagea, S., and Galloway, A. (1998). Intravascular-catheter-related infections. Lancet 351, 1738-1739.

Pastar, I., Stojadinovic, O., Yin, N.C., Ramirez, H., Nusbaum, A.G., Sawaya, A., Patel, S.B., Khalid, L., Isseroff, R.R., and Tomic-Canic, M. (2014). Epithelialization in Wound Healing: A Comprehensive Review. Adv Wound Care 3, 445-464.

Percival, S.L., Suleman, L., Vuotto, C., and Donelli, G. (2015). Healthcare-associated infections, medical devices and biofilms: risk, tolerance and control. J Med Microbiol 64, 323-334.

Rakonjac, J., Bennett, N.J., Spagnuolo, J., Gagic, D., and Russel, M. (2011). Filamentous bacteriophage: biology, phage display and nanotechnology applications. Curr Issues Mol Biol 13, 51-76. 
Rice, S.A., Tan, C.H., Mikkelsen, P.J., Kung, V., Woo, J., Tay, M., Hauser, A., McDougald, D., Webb, J.S., and Kjelleberg, S. (2009). The biofilm life cycle and virulence of Pseudomonas aeruginosa are dependent on a filamentous prophage. ISME J 3, 271-282.

Roux, S., Krupovic, M., Daly, R.A., Borges, A.L., Nayfach, S., Schulz, F., Sharrar, A., Matheus Carnevali, P.B., Cheng, J.-F., Ivanova, N.N., et al. (2019). Cryptic inoviruses revealed as pervasive in bacteria and archaea across Earth's biomes. Nat Microbiol.

Roy, S., Elgharably, H., Sinha, M., Ganesh, K., Chaney, S., Mann, E., Miller, C., Khanna, S., Bergdall, V.K., Powell, H.M., et al. (2014). Mixed-species biofilm compromises wound healing by disrupting epidermal barrier function. J Pathol 233, 331-343.

Scali, C., and Kunimoto, B. (2013). An update on chronic wounds and the role of biofilms. J Cutan Med Surg 17, 371-376.

Schindelin, J., Arganda-Carreras, I., Frise, E., Kaynig, V., Longair, M., Pietzsch, T., Preibisch, S., Rueden, C., Saalfeld, S., Schmid, B., et al. (2012). Fiji: an open-source platform for biological-image analysis. Nat Methods 9, 676-682.

Secor, P.R., Jennings, L.K., Michaels, L.A., Sweere, J.M., Singh, P.K., Parks, W.C., and Bollyky, P.L. (2015a). Biofilm assembly becomes crystal clear - filamentous bacteriophage organize the Pseudomonas aeruginosa biofilm matrix into a liquid crystal. Microb Cell Fact 3, 49-52.

Secor, P.R., Michaels, L.A., Ratjen, A., Jennings, L.K., and Singh, P.K. (2018). Entropically driven aggregation of bacteria by host polymers promotes antibiotic tolerance in Pseudomonas aeruginosa. Proc Natl Acad Sci U S A.

Secor, P.R., Michaels, L.A., Smigiel, K.S., Rohani, M.G., Jennings, L.K., Hisert, K.B., Arrigoni, A., Braun, K.R., Birkland, T.P., Lai, Y., et al. (2017). Filamentous Bacteriophage Produced by Pseudomonas aeruginosa Alters the Inflammatory Response and Promotes Noninvasive Infection In Vivo. Infect Immun 85.

Secor, P.R., Sweere, J.M., Michaels, L.A., Malkovskiy, A.V., Lazzareschi, D., Katznelson, E., Rajadas, J., Birnbaum, M.E., Arrigoni, A., Braun, K.R., et al. (2015b). Filamentous Bacteriophage Promote Biofilm Assembly and Function. Cell Host Microbe 18, 549-559.

Sen, C.K., Gordillo, G.M., Roy, S., Kirsner, R., Lambert, L., Hunt, T.K., Gottrup, F., Gurtner, G.C., and Longaker, M.T. (2009). Human skin wounds: a major and snowballing threat to public health and the economy. Wound Repair Regen 17, 763-771.

Sivamani, K., Garcia, M.S., and Rivkah Isseroff, R. (2007). Wound re-epithelialization: modulating keratinocyte migration in wound healing. Front Biosci 12, 2849-2868.

Sweere, J.M., Ishak, H., Sunkari, V., Bach, M.S., Manasherob, R., Yadava, K., Ruppert, S.M., Sen, C.K., Balaji, S., Keswani, S.G., et al. (2019a). The Immune Response to Chronic Pseudomonas aeruginosa Wound Infection in Immunocompetent Mice. Adv Wound Care.

Sweere, J.M., Van Belleghem, J.D., Ishak, H., Bach, M.S., Popescu, M., Sunkari, V., Kaber, G., Manasherob, R., Suh, G.A., Cao, X., et al. (2019b). Bacteriophage trigger antiviral immunity and prevent clearance of bacterial infection. Science 363.

Tacconelli, E., Carrara, E., Savoldi, A., Harbarth, S., Mendelson, M., Monnet, D.L., Pulcini, C., Kahlmeter, G., Kluytmans, J., Carmeli, Y., et al. (2018). Discovery, research, and development 
of new antibiotics: the WHO priority list of antibiotic-resistant bacteria and tuberculosis. Lancet Infect Dis 18, 318-327.

Tredget, E.E., Shankowsky, H.A., Rennie, R., Burrell, R.E., and Logsetty, S. (2004). Pseudomonas infections in the thermally injured patient. Burns 30, 3-26.

Tu, C.-L., Celli, A., Mauro, T., and Chang, W. (2019). Calcium-Sensing Receptor Regulates Epidermal Intracellular Ca2+ Signaling and Re-Epithelialization after Wounding. J Invest Dermatol 139, 919-929.

Van Belleghem, J.D., Clement, F., Merabishvili, M., Lavigne, R., and Vaneechoutte, M. (2017). Pro- and anti-inflammatory responses of peripheral blood mononuclear cells induced by Staphylococcus aureus and Pseudomonas aeruginosa phages. Sci Rep 7, 8004.

Van Belleghem, J.D., Dąbrowska, K., Vaneechoutte, M., Barr, J.J., and Bollyky, P.L. (2018). Interactions between Bacteriophage, Bacteria, and the Mammalian Immune System. Viruses 11. Wang, X., Ge, J., Tredget, E.E., and Wu, Y. (2013). The mouse excisional wound splinting model, including applications for stem cell transplantation. Nat Protoc 8, 302-309.

Watters, C., DeLeon, K., Trivedi, U., Griswold, J.A., Lyte, M., Hampel, K.J., Wargo, M.J., and Rumbaugh, K.P. (2013). Pseudomonas aeruginosa biofilms perturb wound resolution and antibiotic tolerance in diabetic mice. Med Microbiol Immunol 202, 131-141.

Williams, H., Campbell, L., Crompton, R.A., Singh, G., McHugh, B.J., Davidson, D.J., McBain, A.J., Cruickshank, S.M., and Hardman, M.J. (2018). Microbial Host Interactions and Impaired Wound Healing in Mice and Humans: Defining a Role for BD14 and NOD2. J Invest Dermatol 138, 2264-2274.

Zhao, G., Usui, M.L., Lippman, S.I., James, G.A., Stewart, P.S., Fleckman, P., and Olerud, J.E. (2013). Biofilms and Inflammation in Chronic Wounds. Adv Wound Care 2, 389-399.

Zhao, G., Usui, M.L., Underwood, R.A., Singh, P.K., James, G.A., Stewart, P.S., Fleckman, P., and Olerud, J.E. (2012). Time course study of delayed wound healing in a biofilm-challenged diabetic mouse model. Wound Repair Regen 20, 342-352. 